\title{
National Immunoglobulin replacement Expert Committee recommendations
}

\begin{abstract}
The use of immunoglobulin therapy has grown steadily over the past 3 decades, mainly due to the increased awareness and expansion of indications. This limited resource is now shared between patients with immunodeficiency disorders who need it as replacement therapy, and individuals who suffer a variety of autoimmune or inflammatory disorders. While alternative therapies exist for the latter diseases, immunodeficient patients are dependent upon this treatment for life. Due to the long-term cost burden of this treatment on healthcare systems, healthcare providers have attempted to moderate its use but are frequently seeking evidence from experts in the field. This raised the critical need for recommendations from experts. To this end, a group a Canadian immunologists representing all regions of the country have formed a panel, the National Immunoglobulin replacement Expert Committee (NIGEC), and formulated a set of unanimously agreed upon recommendations for the use of immunoglobulin replacement in primary immunodeficiency.
\end{abstract}

\section{Consensus among experts}

1. Children and adults with a suspected immunodeficiency should be referred to an immunologist with expertise in the field of primary immunodeficiency ("expert" in PID).

2. Ideally, this should be carried out in an academic centre with the capability of performing specialized diagnostic tests for immunodeficiency. Management should be performed by a specialized team including physicians, nurses, and allied health care providers.

3. Ideally, replacement with IVIG or SCIG, changes in dosage, changes in product or its mode of delivery should be initiated directly by "experts" or in consultation with an "expert".

4. Diagnosis of antibody deficiency should be evaluated and ideally confirmed using an appropriate combination of the following tests:
A. Serum immunoglobulin levels.
B. Antibody formation in response to vaccination and the ability to sustain protective levels.
C. Immunophenotyping including enumeration of memory and naïve $\mathrm{B}$ and $\mathrm{T}$ cells.
D. In-vitro mitogenic responses.
E. Assessment of TRECs and TCR-V $\beta$ (where available).
F. Genetic analysis.

5. IVIG and SCIG are similarly efficacious in preventing infections and can therefore be used interchangeably.

6. IVIG and SCIG deliver IgG differently resulting in different pharmacokinetic properties. An "expert" should tailor therapy to what is most suitable for each patient.

7. After a thorough discussion with patients on the benefits and challenges associated with IVIG or SCIG, their preference should be considered when deciding therapy.

8. We recommend the establishment of a national database based on post marketing surveillance to capture adverse reactions to all immunoglobulin products.

9. Immunoglobulin should not be dispensed as a generic product. Patients requiring ongoing infusions should be maintained on a consistent product that is best tolerated by the patient.

10. Consistent national practices should be developed for monitoring patients that are receiving SCIG self-infusions. 
11. We recommend flexible systems of transition from one modality of immunoglobulin administration to another (from IVIG to SCIG or the reverse), to accommodate changing patient needs across the lifespan.

12. Replacement should be at a dose of $400-600 \mathrm{mg} / \mathrm{kg}$ given once every 3-4 weeks for IVIG, or $100-150 \mathrm{mg} / \mathrm{kg}$ per week for SCIG, doses or frequency to be adjusted by experts according to desired trough levels and individual patient clinical needs. Infrequently, patients who metabolize exogenous IgG faster, leading to a shorter half-life, might require more frequent administration.

13. Serum IgG trough levels should exceed $500 \mathrm{mg} / \mathrm{dL}$, and ideally more than $700 \mathrm{mg} / \mathrm{dL}$. Levels should be kept in the normal range per age or increased to obtain optimal clinical response.

14. When serum IgG trough levels drop below protective levels while on SCIG replacement, it is recommended to either transiently switch to IVIG or increase frequency and or dosage of SCIG.

\author{
Stephen Betschel ${ }^{a}$ \\ Peter Dent ${ }^{\mathrm{b}}$ \\ Elie Haddad $^{\mathrm{c}}$ \\ Alison Haynes ${ }^{d}$ \\ Thomas Issekutz ${ }^{\mathrm{e}}$ \\ Bruce Mazer $^{f}$ \\ Brenda Reid $^{g}$ \\ Chaim M. Roifman ${ }^{\mathrm{g}, \mathrm{h} \text { * }}$ \\ Bob Schellenberg ${ }^{i}$ \\ Gordon Sussman ${ }^{\mathrm{j}}$ \\ Stuart Turvey ${ }^{k}$ \\ Susan Waserman'
}

a. Division of Clinical Immunology and Allergy, St. Michael's Hospital, University of Toronto, Toronto, ON

b. Department of Pediatrics, McMaster Children's Hospital, Hamilton, ON

c. Division of Clinical Immunology \& Rheumatology, Pediatric Department, CHU Sainte-Justine, University of Montreal, Montreal, QC

d. Department of Pediatrics, The Janeway Children's Hospital, Memorial University of Newfoundland, St. John's, NL

e. Division of Immunology, IWK Health Centre, Dalhousie University, Halifax, NS

f. Division of Pediatric Allergy and Immunology, Department of Pediatrics, Montreal Children's Hospital, McGill University Health Center, Montreal, QC

g. Division of Immunology and Allergy, Department of Pediatrics, The Hospital for Sick Children, University of Toronto, Toronto, ON

h. Canadian Centre for Primary Immunodeficiency, The Hospital for Sick Children, University of Toronto, Toronto, ON

i. Division of Allergy and Clinical Immunology, Department of Medicine, University of British Columbia, Vancouver, BC

j. Clinical Immunology and Allergy, Faculty of Medicine, University of Toronto, Toronto, ON

k. Division of Clinical Immunology and Allergy, Department of Pediatrics, BC Children's Hospital, University of British Columbia, Vancouver, BC

I. Division of Clinical Immunology and Allergy, McMaster University, Hamilton, ON

*Corresponding author: Chaim M. Roifman/chaim.roifman@ sickkids.ca

dx.doi.org/10.14785/lymphosign-2017-0008

Submitted 31 July 2017

Accepted 17 August 2017

Available online 17 August 2017 